\title{
COSMOLOGICAL EVOLUTION IN A BRANE UNIVERSE
}

\author{
DAVID LANGLOIS \\ Institut d'Astrophysique de Paris, \\ Centre National de la Recherche Scientifique, \\ 98bis Boulevard Arago, 75014 Paris, France \\ and \\ Département d'Astrophysique Relativiste et de Cosmologie, \\ Centre National de la Recherche Scientifique, \\ Observatoire de Paris, 92195 Meudon Cedex, France
}

\begin{abstract}
The idea of extra-dimensions has recently gone through a renewal with the hypothesis, suggested by recent developments in string theory, that ordinary matter is confined to a sub-space, called brane, embedded in a higher dimensional spacetime. I summarize here some consequences in cosmology of this type of models. The most remarkable aspect is that the Friedmann laws, which govern the expansion of the Universe, are modified. An important direction of research is the study of cosmological perturbations and the possible signature of extra-dimensions in cosmological observations.
\end{abstract}

\section{Homogeneous brane cosmology}

It has been recently suggested that there could exist extra-dimensions which are not accessible to ordinary matter, in the sense that matter would be confined to a three-dimensional subspace, or brane, within a higher dimensional space or bulk. In this context, a lot of attention has been devoted to cosmology, essentially to five-dimensional models where our Universe would be a hypersurface.

One of the first striking results was that, when solving the five-dimensional Einstein's equations $G_{A B} \equiv R_{A B}-R g_{A B} / 2=\kappa^{2} T_{A B}$, the matter content of the brane appears quadratically $\mathrm{\omega}$ in Friedmann's equations instead of linearly as in standard cosmology. In the case of an empty bulk, one would thus find a cosmological evolution incompatible with our understanding of nucleosynthesis. A way out has been found by applying the Randall-Sundrum idea 2 to cosmology 3 , i.e. considering an Anti-de Sitter bulk spacetime (with a negative cosmological constant $\Lambda$ ) and a tension in the brane. The (assumed) cancellation of $\Lambda$ with the square of the brane tension $\sigma$ leads to the new Friedmann equations Et $^{-1}$

$$
H^{2}=\frac{8 \pi G}{3}\left(\rho+\frac{\rho^{2}}{2 \sigma}\right)
$$

where $H$ is the Hubble parameter in the brane, $\rho$ the cosmological energy density in the brane. And Newton's constant is related to the brane tension by $8 \pi G=$ $\kappa^{4} \sigma / 6$. This equation gives the usual evolution in the low energy regime $\rho \ll \sigma$ and quadratic corrections in the high energy regime $\rho>\sigma$.

\section{Cosmological perturbations in brane cosmology}

The next step is obviously to investigate what will be the influence of extradimensions on the cosmological perturbations and their evolution. Several pioneer- 
ing works have developed formalisms to handle the cosmological perturbations for a brane-universe in a five-dimensional spacetime 6 .

The study of perturbations during a de Sitter phase in the brane is made easier by the fact that the background evolution is rather simple. One can for example compute explicitly the spectrum of gravitational waves generated in a de Sitter phase on the brane $\mathrm{U}$.

For radiation or matter dominated eras, the evolution of perturbations is much more complicated. However, it is possible to rewrite the evolution equations for the cosmological perturbations in the brane in a form very close to the equations of standard cosmology with two types of corrections: a. corrections due to the unconventional evolution of the homogeneous solution (in the high energy regime), which change the background-dependent coefficients of the equations; b. corrections due to the curvature along the fifth dimension, which act as source terms, i.e. 'active seeds', in the evolution equations 6 . This reformulation of the five-dimensional equations makes transparent the way the perturbations due to the fifth dimension could have an impact, from the point of view $g$ a brane observer, for instance on the

cosmic Microwave Background anisotropies 9 , but a quantitative analysis depends on the specific distribution of gravitational waves in the bulk.

\section{References}

1. P. Binétruy, C. Deffayet, D. Langlois, Nucl. Phys. B 565, 269 (2000) hepth/9905012.

2. L. Randall, R. Sundrum, Phys. Rev. Lett. 83, 4690 (1999) hep-th/9906064.

3. C. Csáki, M. Graesser, C. Kolda, J. Terning, Phys. Lett. B462, 34 (1999); J.M. Cline, C. Grojean, G. Servant, Phys. Rev. Lett. 83, 4245 (1999).

4. P. Binétruy, C. Deffayet, U. Ellwanger, D. Langlois, Phys. Lett. B 477, 285 (2000) hep-th/9910219.

5. P. Kraus, JHEP 9912, 011 (1999) hep-th/9910149; T. Shiromizu, K. Maeda, M. Sasaki, Phys. Rev. D 62, 024012 (2000) ggr-qc/9910076]; E. Flanagan, S. Tye, I. Wasserman, Phys. Rev. D 62, 044039 (2000) hep-th/9910498

6. H. Kodama, A. Ishibashi, O. Seto, Phys. Rev. D 62, $064022(2000)$ hepth/0004160; R. Maartens, Phys. Rev. D 62, 084023 (2000) hep-th/0004166; D. Langlois, Phys. Rev. D 62, 126012 (2000) hep-th/0005025; C. van de Bruck, M. Dorca, R. Brandenberger, A. Lukas, Phys. Rev. D 62, 123515 (2000) hep-th/0005032; K. Koyama, J. Soda, Phys. Rev. D 62, 123502 (2000) hep-th/0005239; N. Deruelle, T. Dolezel, J. Katz, hep-th/0010215,

7. D. Langlois, R. Maartens, D. Wands, Phys. Lett. B 489, 259 (2000) hepth/0006007

8. D. Langlois, "Evolution of cosmological perturbations in a brane-universe", hep-th/0010063, to appear in Phys. Rev. Lett.

9. D. Langlois, R. Maartens, M. Sasaki, D. Wands, "Large-scale cosmological perturbations on the brane", hep-th/0012044. 